\title{
Identification of a novel compound heterozygous mutation of the CYP21A2 gene causing 21-hydroxylase deficiency in a Chinese pedigree
}

\author{
JIA LIU ${ }^{1-3}$, XIUJUAN ZHANG ${ }^{1-3}$, HAIQING ZHANG ${ }^{1-3}$, LI FANG ${ }^{2,3}$, \\ JIN XU ${ }^{1-3}$, QINGBO GUAN ${ }^{1-3}$ and CHAO XU ${ }^{1-3}$ \\ ${ }^{1}$ Department of Endocrinology, Shandong Provincial Hospital Affiliated to Shandong University; \\ ${ }^{2}$ Shandong Clinical Medical Center of Endocrinology and Metabolism; ${ }^{3}$ Institute of Endocrinology and Metabolism, \\ Shandong Academy of Clinical Medicine, Jinan, Shandong 250021, P.R. China
}

Received May 11, 2017; Accepted November 16, 2017

DOI: $10.3892 / \mathrm{mmr} .2018 .8391$

\begin{abstract}
Hydroxylase deficiency (21-OHD) is the most common cause of congenital adrenal hyperplasia. Inherited in an autosomal recessive manner, 21-OHD is caused by mutations in the cytochrome $\mathrm{P} 450$ family 21 subfamily A member 2 (CYP21A2) gene. The present study was designed to investigate the genetic characteristics of one Chinese pedigree and to identify the genotype-phenotype association, thereby facilitating the precise diagnosis of 21-OHD at the molecular level. Members of a Chinese family with 21-OHD were screened for mutations in the CYP21A2 gene. Clinical data and biochemical parameters, including androgen and derivatives, were collected. Complete DNA sequencing and multiplex ligation-dependent probe amplification (MLPA) were utilized to analyze the genetic variations in the full-length CYP21A2 gene. A C-T transition located in exon 8 of the CYP21A2 gene, leading to the predicted amino acid residue change from Arg to Trp at codon 342, was identified in the mother and four sisters. Additionally, heterozygous deletion mutations of exons 1, 3, 4, 6 and 7 of paternal origin were detected in the four sisters by MLPA analysis. During the one-year follow-up, the four sisters exhibited symptom improvement following treatment with glucocorticoids, and the proband and one sister successfully conceived. The results of the present study demonstrated that novel compound heterozygous variations in the CYP21A2 gene may be causative agents of 21-OHD, providing insights into the functions of this gene and a more comprehensive understanding of the disorder.
\end{abstract}

Correspondence to: Professor Chao Xu, Department of Endocrinology, Shandong Provincial Hospital Affiliated to Shandong University, 324 Jingwu Road, Jinan, Shandong 250021, P.R. China

E-mail: doctorxuchao@163.com

Key words: 21-hydroxylase deficiency, congenital adrenal hyperplasia, cytochrome P450 family 21 subfamily A member 2, mutation

\section{Introduction}

Congenital adrenal hyperplasia $(\mathrm{CAH})$ is one of the most common inherited metabolic disorders, and is associated with substantial morbidity and mortality in affected children and adults (1). Steroid 21-hydroxylase deficiency (21-OHD), the most common variant of $\mathrm{CAH}$, accounts for $\sim 90 \%$ of $\mathrm{CAH}$ cases (2,3). Patients most commonly present with symptoms associated with different extents of androgen accumulation, a hallmark of $\mathrm{CAH}$, with or without aldosterone deficiency $(1,3)$. Based on the broad spectrum of presentations, $\mathrm{CAH}$ has been divided into two categories: Classical, which is represented by salt wasting ( $\mathrm{SW} ;<2 \%$ 21-OH activity) and simple virilizing (SV) forms (an increase of $\sim 1-2 \%$ in $21-\mathrm{OH}$ activity compared with SW); and non-classical (NC; 20-50\% 21-OH activity).

Inherited in an autosomal recessive manner, 21-OHD is caused by mutations in the cytochrome P450 family 21 subfamily A member 2 (CYP21A2) gene, which is located on chromosome $6 \mathrm{p} 21.3, \sim 3.1 \mathrm{~kb}$ from the highly homologous CYP21A1 pseudogene (CYP21A1P) (4-7). At present, $>200$ CYP21A2-inactivating mutations are listed in the Human Gene Mutation Database (HGMD; www.hgmd.org; Table I). The majority of these mutations are missense and nonsense mutations. However, splice site mutations, deletions and insertions, in addition to other types of mutations, have been detected. Due to complex mutation types and the variety of clinical presentations, identification of the genotype it is required to correlate with the phenotype.

In the present study, molecular genetic analysis of the CYP21A2 gene was performed in a patient with 21-OHD and other family members. A novel compound heterozygous mutation, not previously described, was identified and confirmed to be responsible for 21-OHD in this Chinese pedigree. Managed with hormone replacement therapy, the proband and the sister of the proband succeeded in conceiving following 6 months of treatment with dexamethasone. The results of the present study provided novel information clarifying the $\mathrm{CAH}$ genotype-phenotype association, offering evidence for patient counseling and improved prenatal diagnosis. 


\section{Subjects and methods}

Subjects. The present study was performed according to the human research guidelines in the Declaration of Helsinki. The research protocol was approved by the institutional review board of Shandong Provincial Hospital, and written informed consent was obtained from all subjects. All family members were examined and blood was collected between September 2015-November 2015 for genetic analysis. Subjects received a one-year follow-up.

Pedigree. The pedigree, a two-generation family consisting of six individuals, including the patient, and the father, mother and three elder sisters of the patient, was referred to Shandong Provincial Hospital (Jinan, China). The pedigree chart is displayed in Fig. 1.

Examinations. Circadian rhythm analysis of blood cortisol and a low-dose dexamethasone suppression test were performed. A computed tomography (CT) scan of the adrenal gland and magnetic resonance imaging of pituitary gland were performed. Ultrasonic examination of gynecopathy was also accomplished.

DNA extraction, polymerase chain reaction (PCR) analysis and sequencing. Peripheral blood $(4 \mathrm{ml})$ was collected from all family members following $12 \mathrm{~h}$ of fasting using EDTA. DNA was extracted from white blood cells using a DP304-03 genomic DNA kit (Tiangen Biotech Co., Ltd., Beijing, China). PCR was performed as previously reported using an Eppendorf Master Cycler 5333 system (Eppendorf, Hamburg, Germany) (8). A total of four pairs of primers were used to amplify CYP21A2 exons 1-3, 1-6, 3-10, and 6-10 without amplifying the neighboring CYP21A1P gene (Table II). For gene sequencing, capillary electrophoresis was implemented using the ABI genetic analyzer 3,130xl (Thermo Fisher Scientific, Inc., Waltham, MA, USA) (9). The results were analyzed using Mutation Surveyor_5.0-UG001 (www.softgenetics.com).

Multiplex ligation-dependent probe amplification (MLPA) analysis. MLPA analysis was performed in all cases to identify large gene deletions and conversions in the CYP21 A2 gene using the SALSA MLPA kit P050CAH probemix/P050-C1-0714 CAH-v31 (MRC-Holland BV, Amsterdam, The Netherlands). Hybridization, ligation, and amplification were performed according to the manufacturer's protocol. Amplification products were detected using an ABI 3,130xl Genetic Analyzer (Thermo Fisher Scientific, Inc.) with LIZ500 as an internal size standard. The raw data were analyzed using Coffalyser v9.4 software (MRC-Holland BV).

Analysis of CYP21A2 gene mutations. The whole coding region of the CYP21A2 gene, covering the intron-exon boundaries, was directly sequenced using a dye terminator cycle-sequencing system on an ABI genetic analyzer 3,130xl (Thermo Fisher Scientific, Inc.). The resulting sequences were compared to the corresponding wild-type sequences of CYP21A2 using Auto Assembler software (version 2.0; PerkinElmer, Inc., Waltham, MA, USA). The +1 numbering of CYP21A2 genomic DNA corresponds to the A of the ATG
Table I. Various types of CYP21A2 gene mutation, according to the Human Gene Mutation Database.

\begin{tabular}{lr}
\hline Mutation type & No. \\
\hline Missense/nonsense & 172 \\
Splicing & 18 \\
Regulatory & 2 \\
Small deletions & 22 \\
Small insertions & 13 \\
Small indels & 3 \\
Gross deletions & 13 \\
Gross insertions & 3 \\
Complex rearrangements & 39 \\
Repeat variations & 0
\end{tabular}

CYP21A2, cytochrome P450 family 21 subfamily A member 2.

translation initiation codon. The mutations were designated using the recommendations of the Nomenclature Working Group (10,11), in which the genomic and cDNA sequence positions were designated by the prefixes g. and c., respectively.

\section{Results}

Patient history and clinical evaluation. The proband, a 28-year-old Chinese female, was referred to the Shandong Provincial Hospital. The proband presented with 14 years of enlarged prominentia laryngea and 7 years of irregular menstruation. At age 14, the proband was tall compared with her peers, with prominentia laryngea enlargement and excessive pubic hair growth, although no menstrual cramps. However, by the age of 16 , the proband was of below-average height and at age 21 , the proband began to experience abnormal menstruation. The proband did not present to the clinic until the age of 23. Due to the non-classical symptoms and lack of genetic identification, the diagnosis was not accurately made prior to referral to the Shandong Provincial Hospital. Notably, three sisters of the proband experienced similar symptoms, although they did not consult a doctor. At the time of examination, the proband was $153 \mathrm{~cm}$ in height and $60 \mathrm{~kg}$ in weight, with a blood pressure of 111/70 mmHg. Physical examination revealed excessive hair growth (including facial, axillary, leg and pubic hair), acne, prominentia laryngea, breast hypoplasia, outer lip hypertrophy, clitoral hypertrophy, and oligomenorrhea. Biochemical testing (Table III) revealed elevated testosterone, $17 \alpha$-hydroxyprogesterone and androstenedione levels, normal dehydroepiandrosterone and aldosterone levels, and normal sodium and potassium levels. Similar biochemical abnormalities were detected in the eldest sister of the proband (data not shown). Circadian rhythm analysis of blood cortisol and a low-dose dexamethasone suppression test demonstrated that the circadian rhythm of cortisol secretion was lost and was not suppressed by low-dose dexamethasone (data not shown). A computed tomography (CT) scan of the adrenal gland revealed bilateral hyperplasia (Fig. 2), and magnetic resonance imaging revealed an abnormal pituitary gland. Ultrasonic examination of gynecopathy indicated 
Table II. Four pairs of primers of CYP21A2.

\begin{tabular}{llcccc}
\hline No & \multicolumn{1}{c}{ Sequence (5'-3') } & Base position & Exon & Fragment size (bp) & Annealing temperature \\
\hline I & $\begin{array}{l}\text { F: TCGGTGGGAGGGTACCTGAAG } \\
\text { R: AGCTGCATCTCCACGATGTGA }\end{array}$ & $-20-1,518$ & $1 \rightarrow 6$ & 1,522 & 58 \\
II & $\begin{array}{l}\text { F: CCTGTCCTTGGGAGACTACTC } \\
\text { R: GGGACATCCGGVTTGA }\end{array}$ & $918-3,189$ & $3 \rightarrow 10$ & 2,370 & 52 \\
III & $\begin{array}{l}\text { F: AGGGATCACATCGTGGAGA } \\
\text { R: GGGACATCCGGCTTTGA }\end{array}$ & $1493-3,189$ & $6 \rightarrow 10$ & 1,697 & 56 \\
IV & $\begin{array}{l}\text { F: TTTTGTCTTCAGGCGATTC } \\
\text { R: GGGAGTAGTCTCCCAGGAC }\end{array}$ & $-310-940$ & $1 \rightarrow 3$ & 1,150 & 52 \\
\hline
\end{tabular}

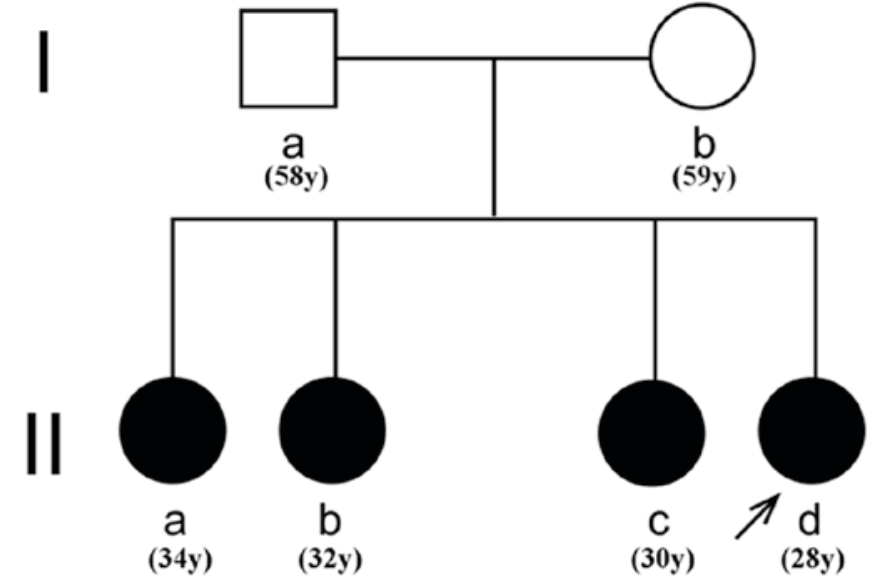

Figure 1. Pedigree of a Chinese family affected by 21-hydroxylase deficiency. Squares, male; circles, female; solid, affected; open, unaffected. Arrow indicates proband. I and II indicate the generation number.

decreased uterus volume. The chromosomal karyotype of the proband was $46 \mathrm{XX}$. The three sisters experienced similar symptoms.

Mutation analysis. The DNA fragments of the parents and four sisters were sequenced in the forward and reverse orientations and the resulting sequences were compared with the corresponding wild-type sequences of CYP21A2. A missense mutation, C-T (Fig. 3; indicated by an arrow), at nucleotide 1,024 in exon 8 of the CYP21A2 gene, which led to the predicted amino acid residue change from Arg to Trp at codon342, was identified in the mother, the proband and the three sisters of the proband. In the present study a previously reported point mutations in exons based on the HGMD, including the missense variant identified in the present study was summarized (Fig. 4). Additionally, MLPA analysis was performed to determine large gene deletions. As indicated by the red arrows in Fig. 5, a 35-50\% reduction in the relative peak height of the amplification product of exons 1, 3, 4, 6 and 7 was identified in the father, the proband as well as the three sisters (data not shown), indicating paternal heterozygous deletion mutations. Therefore, the four sisters in the present study all exhibited a compound heterozygous mutation in the CYP21A2 gene, including a missense mutation c.1024C>T (p. Arg342Trp) in exon 8 of maternal origin and heterozygous deletion mutations of exons 1, 3, 4, 6 and 7 of paternal origin (Fig. 6).

Bioinformatics analysis. The missense mutation c.1024C $>\mathrm{T}$ (p. Arg342Trp) in exon 8 results in an alteration in amino acid composition by replacing an arginine with a tryptophan, thereby affecting the function of the protein. Heterozygous deletion mutations in exons 1, 3, 4, 6 and 7 lead to mutations that alter a codon specific for one amino acid to one specific for another amino acid, thus affecting the amino acid composition and the polypeptide chain sequence. Since the polypeptide chains lose their appropriate primary amino sequence, they fail to form functional proteins. Therefore, these mutations may severely affect protein function and induce a series of pathologies.

Therapy and 1-year-follow-up. Following 4 months of treatment with dexamethasone, the elder sister of the proband conceived. The proband received periodic review, following the recommendations of a doctor. Following treatment, the results gradually improved, as testosterone, adrenocorticotropic hormone (ACTH) and cortisol levels were within normal range (Table II). Following 6 months of treatment with dexamethasone, the proband conceived. The results of the present study suggested that an annual clinical follow-up maybe recommended to observe the recovery of the patient.

\section{Discussion}

In the present study, it was demonstrated that a compound heterozygous mutation, a missense mutation c.1024C $>$ T (p. Arg342Trp) and heterozygous deletion mutations of exons 1, 3, 4, 6 and 7 in the CYP21A2 gene may serve an important role in a Chinese pedigree of four sisters affected by 21-OHD. To the best of the authors' knowledge, this compound heterozygous mutation has not yet been reported elsewhere. The confirmation of a CAH diagnosis by genetic analysis is important to correlate genotype with phenotype. In addition, the patient presented with SV-CAH, and a detailed molecular analysis may provide useful insights into the functions of the CYP21A2 gene.

The 21-OH locus includes a real gene, functional CYP21A2, which encodes a protein of 495 amino acids, and the non-functional CYP21A1P, which is a pseudogene and hence does not 
Table III. Biochemical parameter analysis in the proband.

\begin{tabular}{lcc}
\hline Parameter & Results & Reference range \\
\hline Aldosterone, pmol/l & 203.13 & $40-310$ \\
Follicle stimulating hormone, mIU/ml & 5.61 & $1.27-19.26$ \\
Luteinizing hormone, $\mathrm{mIU} / \mathrm{ml}$ & 2.76 & $1.24-8.62$ \\
Progesterone, $\mathrm{ng} / \mathrm{ml}$ & 6.13 & $0.1-0.2$ \\
Testosterone, $\mathrm{ng} / \mathrm{ml}$ & 1.59 & $0-0.75$ \\
Testosterone, ng/ml (post-treatment) & 0.22 & $0-0.75$ \\
17a-Hydroxyprogesterone, ng/ml & 8.97 & $0.07-1.53$ \\
Dehydroepiandrosterone, $\mu \mathrm{g} / \mathrm{dl}$ & 271.70 & $98.8-340$ \\
Androstenedione, nmol/l & 8.25 & $4-6.6$ \\
Potassium, mmol/l & 3.70 & $3.5-5.5$ \\
Sodium, mmol/l & 139.00 & $135-155$ \\
Adrenocorticotropic hormone (8:00), pg/ml & 118.20 & $7.2-63.3$ \\
Cortisol (8:00), nmol/l & 562.80 & $171-536$ \\
Adrenocorticotropic hormone (8:00), pg/ml (post-treatment) & 26.60 & $7.2-63.3$ \\
Cortisol (8:00), nmol/l (post-treatment) & 510.70 & $171-536$
\end{tabular}

A

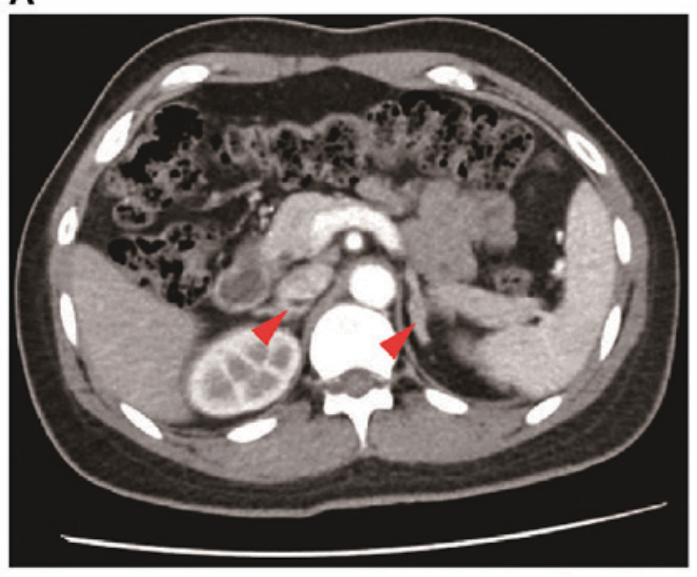

B

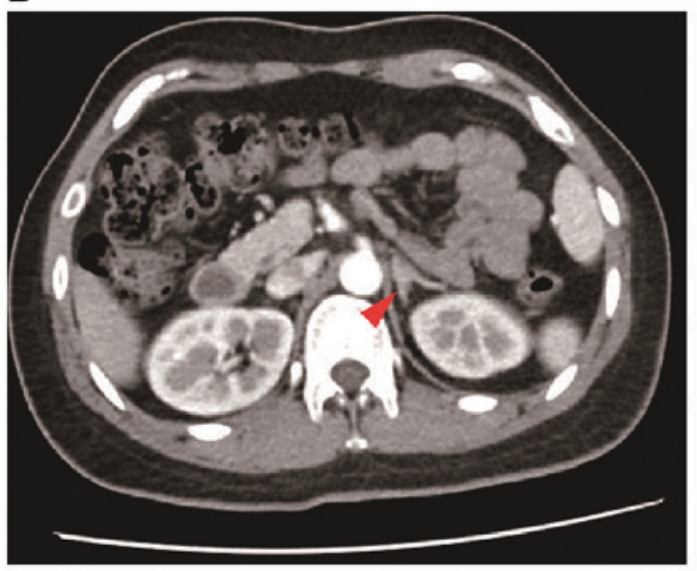

Figure 2. Transverse CT scan sections of the proband's adrenal gland, (A) at the level of T12 and (B) 3 mm below A. Red arrows indicate bilateral adrenal hyperplasia. CT, computed tomography.

code for a protein. CYP21A2 and CYP21A1 Peach consist of 10 exons and 9 introns, with $98 \%$ homology among exons and $96 \%$ homology among introns (5). Due to their high homology, CYP21A2 and CYP21A1P are prone to complex and diverse genetic recombinations, which is one of the reasons for the relatively high incidence of mutations. A total of $~ 95 \%$ of CYP21A2 mutations result from intergenic recombination between CYP21A2 and the CYP21A1P pseudogene. The complete deletion of neighboring complement $\mathrm{C} 4 \mathrm{~B}$ and a net deletion of CYP21A1, or apparent gene conversion events that transfer deleterious mutations normally present in CYP21A1P to CYP21A2, may occur due to unequal crossing over during meiosis (12). These defects or repeated fragments stimulate RCCX unit homologous genes to form different chimeras (13).

The sequence variants identified in the present study are hypothesized to be disease-causing mutations in this Chinese pedigree. The missense mutation c.1024C $>$ T (p. Arg342Trp) detected in the mother and four sisters is expected to exert a substantial impact on the structure and function of the CYP21A2 protein due to the replacement of an arginine with a tryptophan. Arginine residues that exist in the cytochrome P450 enzymes of different mammalians are highly conserved in the family and are important for enzymatic activity (14). Arginine is a positively-charged polar amino acid with a long hydrophobic side chain, whereas tryptophan is a non-polar aromatic amino acid and thus is not conducive to the formation of an $\alpha$-helix. In the present study, the patient and mother of the patient presented with the same hybrid genetic mutations in CYP21A2, indicating that these mutations are of maternal inheritance. The loss of the larger pieces of the gene adversely influences its functions, altering a number of essential amino acids $(15,16)$. In the present study, heterozygous deletion mutations of exons 1, 3, 4, 6 and 7 of paternal origin were additionally detected, leading to early 
A C site was normal
C G T

la

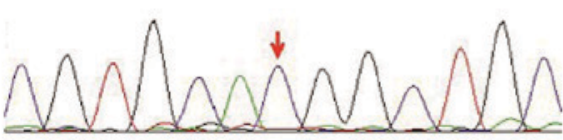

C

c. $1024 \mathrm{C}>\mathrm{T}, \mathrm{CGG} \rightarrow \mathrm{TGG}$

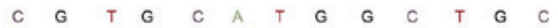

Ila

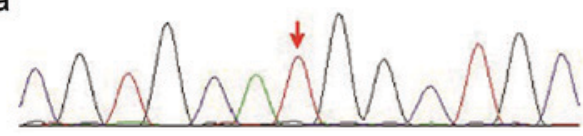

E

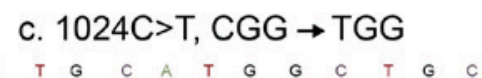

IIc

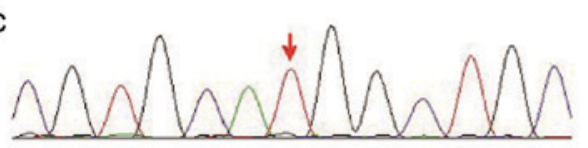

B

Ib

c. $1024 \mathrm{C}>\mathrm{T}, \mathrm{CGG} \rightarrow \mathrm{TGG}$
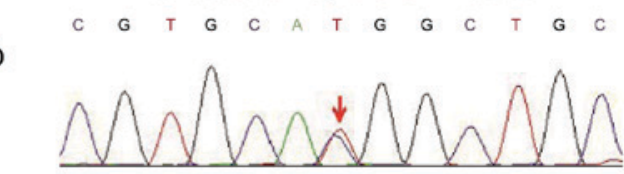

D

c. $1024 \mathrm{C}>\mathrm{T}, \mathrm{CGG} \rightarrow \mathrm{TGG}$
Ilb
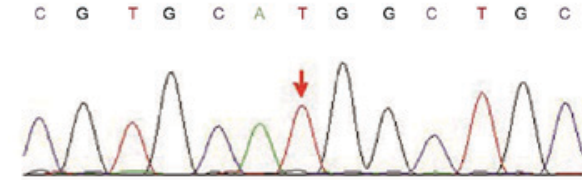

$\mathbf{F}$

c. $1024 \mathrm{C}>\mathrm{T}, \mathrm{CGG} \rightarrow \mathrm{TGG}$

Ild
$C G \quad T \quad G \quad C \quad A \quad T \quad G \quad G \quad C \quad T \quad G \quad C$

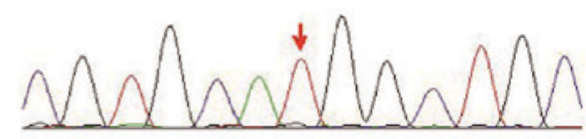

Figure 3. A mutation in the cytochrome P450 family 21 subfamily A member 2 gene was identified in this Chinese pedigree. The mutation, Arg342Trp is caused by a C-T transition at nucleotide 1,024 of exon 8 (red arrows). Data are presented for (A) the father; (B) the mother; (C) the eldest sister; (D) the second sister; (E) the third sister; and (F) the proband. I, generation 1; II, generation 2.

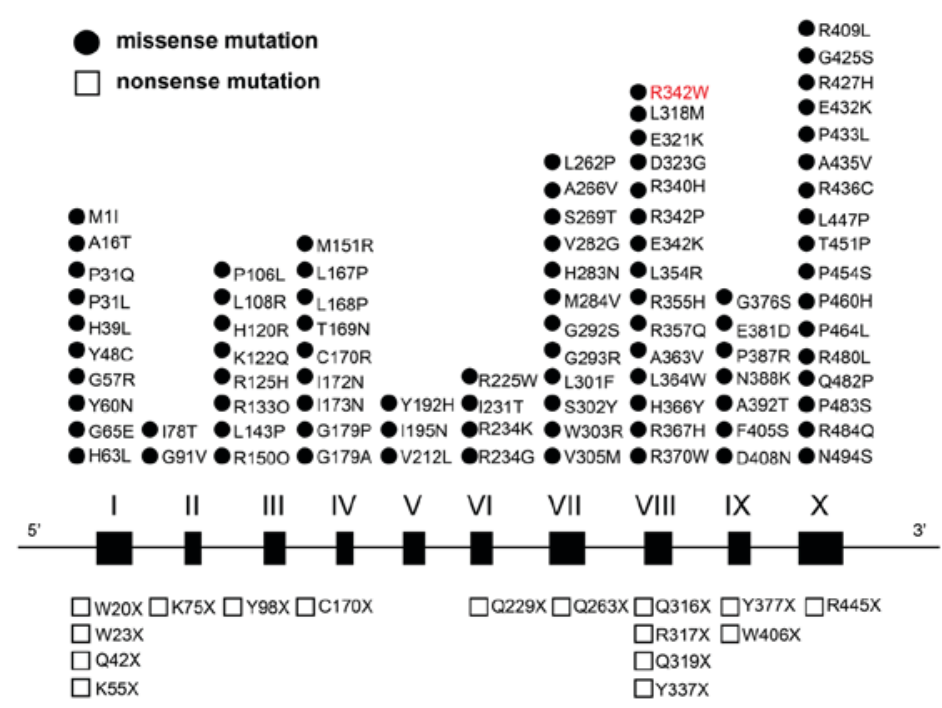

Figure 4. Point mutations, including missense mutation and nonsense mutations, located in different exons of the cytochrome P450 family 21 subfamily A member 2 gene. Circles represent missense mutations; squares represent nonsense mutations. The red font indicates the newly-identified missense mutation in exon 8 in the present study.

termination of protein translation and the creation of a truncated protein. The remaining amino acids were unable to form the four important structural domains, thereby deactivating CYP21A2.

Clinical manifestations of 21-OHD are complex and various. 21-OHD is primarily characterized by cortisol deficiency and the increased accumulation of progesterone, $17 \alpha$-hydroxyprogesterone, and androgen, with or without aldosterone deficiency $(3,12)$. In general, 21-OHD may be divided into SV, SW and NC types, according to the level of enzyme activity. The SV form is characterized by virilization of the external genitalia in newborn females and by hypocortisolism and precocious pseudopuberty due to reactive androgen overproduction in the two sexes. In addition to hyperandrogenism signs, the SW type manifests with an insufficient secretion of aldosterone, hyponatremia and hyperkalemia. The symptoms of the NC form are variable and patients are asymptomatic at birth, manifesting symptoms that include hirsutism, oligomenorrhea and anovulation later in life. In the present study, the proband presented clitoral hypertrophy, and biochemical tests indicated increased $17 \alpha$-hydroxyprogesterone, ACTH and testosterone. Adrenal CT revealed clear bilateral adrenal hyperplasia. The proband was diagnosed with $\mathrm{CAH}$ with normal blood pressure and no symptoms of salt loss. Therefore, the proband was diagnosed with SV CAH. 

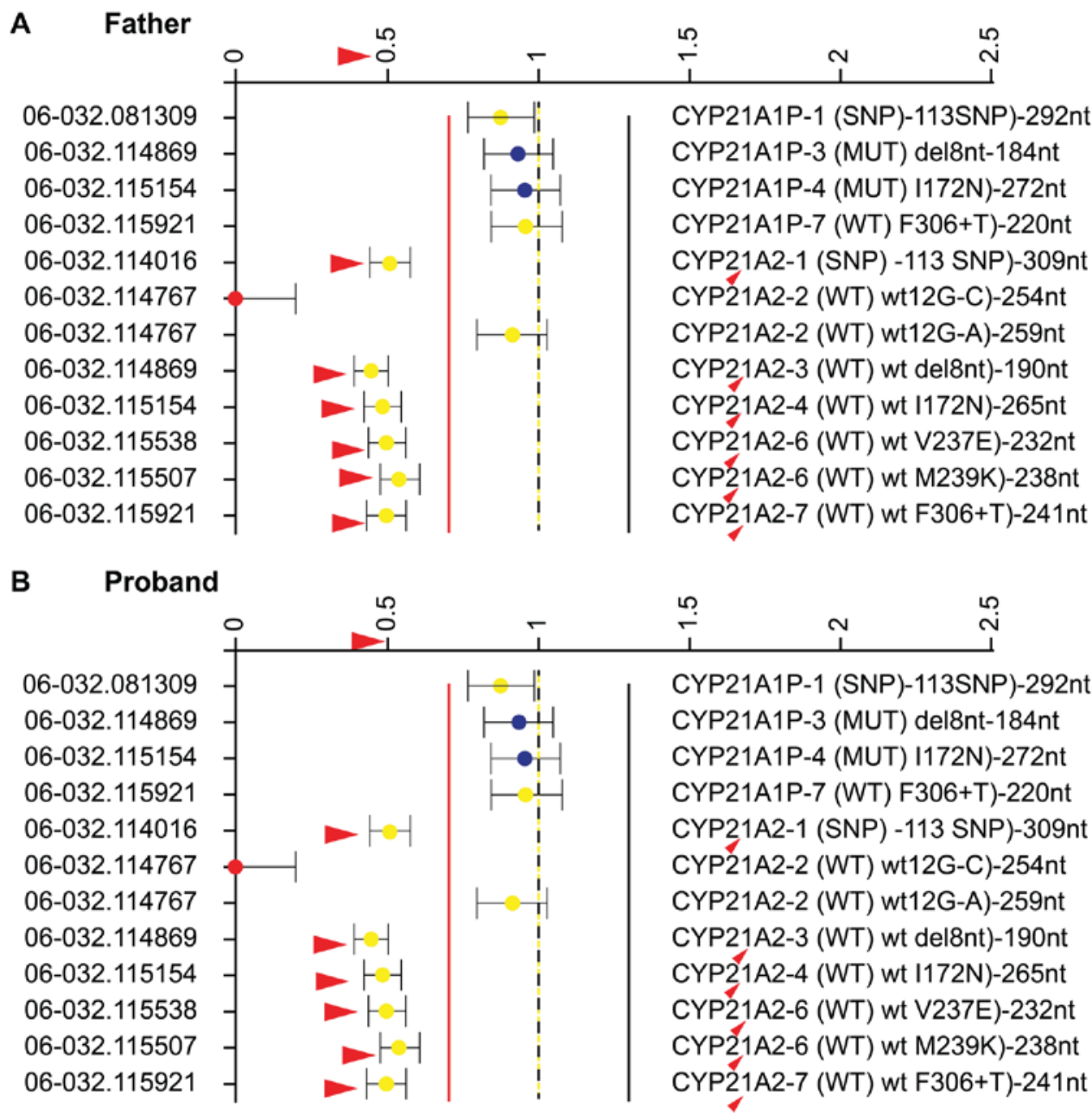

Figure 5. Map view of the multiplex ligation-dependent probe amplification analysis. (A) Relative peak ratios of probes of the father, who exhibited heterozygous deletion mutations of CYP21A2 exons 1,3,4,6 and 7 (red arrows). (B) Relative peak ratios of probes of the proband, who presented with the same heterozygous loss of CYP21A2 exons 1, 3, 4, 6 and 7 (red arrows). CYP21A2, cytochrome P450 family 21 subfamily A member 2; SNP, single-nucleotide polymorphism.

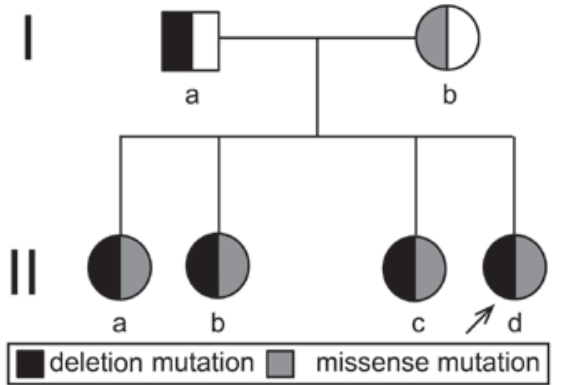

Figure 6. Schematic representation of the cytochrome P450 family 21 subfamily A member 2 gene mutation in a Chinese pedigree. Black, deletion mutation; grey; missense mutation. Arrow indicates proband. I and II indicate the generation number.

The molecular diagnosis of $\mathrm{CAH}$ is complex due to its unusual features. At present, genetic diagnosis of 21-OHD for patients with $\mathrm{CAH}$ is associated with two principal difficulties. CYP21A2 is composed of 10 exons, and each exon is likely to present genetic mutations, loss or amplification of small fragments. Thus, it is recommended that the full-length 21-OHD be sequenced for each patient, which is time-consuming (17). The conventional method used for the detection of a 21-OHD mutation is as follows: A specific primer is designed to amplify the gene segments of CYP21A2, the different amplified fragments are directly sequenced via fragment purification, and the full-length sequence of the CYP21A2 gene as-obtained is used to determine the genetic mutation site (18). The all-gene sequencing method is used to identify the mutation site (19). The second difficulty is the deficiency or amplification of large pieces in the 21-OHD gene of patients with $\mathrm{CAH}$, and such complexity and diversity further complicate the detection. The most commonly used method is Southern imprinting (20), although this is not suitable for a large-scale population or for use in the clinic. In addition, certain conventional methods have been adopted, including long PCR-enzyme digestion analysis, PCR product T-A cloning analysis, and quantitative PCR-based methods for determining CYP21A2 gene copy number $(21,22)$. MLPA maybe used for copy number detection of multiple CYP21A2 gene sites simultaneously (23). This technology combined with gene sequencing is therefore commonly used for CYP21A2 gene diagnosis. Additionally, when a single missing exon is detected by MLPA inspection, it maybe verified by sequencing and by long-segment PCR. 
The phenotype of 21-OHD is associated with its genotype, including point mutations, frame shift mutations and deletion mutations of small DNA fragments. SW-, SV- or NC-type 21-OHD maybe predicted by clinical gene sequencing. Concolino et al (24) demonstrated that the consensus between genotype and SW CAH patients was $81.8 \%$. Baumgartner-Parzer et al (25) reported that the consistency between genotype and clinical phenotype was $80 \%$. However, genotype and clinical phenotype are not always completely consistent, and the same 21-OHD gene mutation in patients with CAH may result in different clinical phenotypes $(26,27)$. New et al $(28)$ reported that certain mutations, including the P30L, I2Gand I172N mutations, were prone to yield different CAH phenotypes. In SW and NC CAH, a phenotype maybe attributed to a genotype. However, in SV $\mathrm{CAH}$, wide phenotypic variability may be observed, particularly with the exon 4 I172N mutation (28). Janjanin et al (29) described five patients with 21-OHD belonging to three generations of the same family (grandmother, parents and two children), whose genotype corresponded to NC CAH. Notably, in spite of one minorCYP21 mutation, the proband presented with the SV form of 21-OHD and required glucocorticoid replacement therapy from the age of 4 years. In the present study, the CYP21A2 gene sequencing of the patient, in addition to the clinical symptoms and gene sequencing results, revealed c.1024C $>\mathrm{T}$ (p. Arg342Trp) and heterozygous deletion mutations, which supported the clinical diagnosis of SV CAH.

The present study provided strong evidence for the presence of a novel compound heterozygous mutation in the CYP21A2 gene that is predicted to result in the partial loss of enzymatic activity of the resulting protein. This mutation caused SV 21-OHD in a Chinese pedigree. The results of the present study provided information for the prenatal diagnosis of $\mathrm{CAH}$ caused by 21-OHD using amniotic cells or chorionic villi. The in vitro expression analysis of residual enzymatic activity may be performed to analyze the functional consequences of this newly identified mutation, thereby proving its relevance to the clinical presentation and analyzing its impact on CYP21A2 function. By investigating the molecular genetic mechanisms of $\mathrm{CAH}$, the identification and diagnosis may be optimized, which has substantial clinical value for diagnosis and genetic counseling of carriers in the family of a patient.

\section{Acknowledgements}

The present study was supported by the grants from the National Natural Sciences Foundation of China (grant nos. 81100593, 81770785, 81370891 and 81670720), special funds for Taishan Scholar Project (grant no. tsqn20161071) and the Provincial key research and development plan (2017GSF18154).

\section{References}

1. Bachelot A, Grouthier V, Courtillot C, Dulon J and Touraine P: Management of endocrine disease: Congenital adrenal hyperplasia due to 21-hydroxylase deficiency: Update in management of adult patients and prenatal treatment. Eur J Endocrinol 176: R167-R181, 2017.

2. Forest MG: Recent advances in the diagnosis and management of congenital adrenal hyperplasia due to 21-hydroxylase deficiency. Hum Reprod Update 10: 469-485, 2004.
3. Speiser PW, Azziz R, Baskin LS, Ghizzoni L, Hensle TW, Merke DP, Meyer-Bahlburg HF, Miller WL, Montori VM, Oberfield SE, et al: Congenital adrenal hyperplasia due to steroid 21-hydroxylase deficiency: An endocrine society clinical practice guideline. J Clin Endocrinol Metab 95: 4133-4160, 2010.

4. White PC, Grossberger D, Onufer BJ, Chaplin DD, New MI, Dupont B and Strominger JL: Two genes encoding steroid 21-hydroxylase are located near the genes encoding the fourth component of complement in man. Proc Natl Acad Sci USA 82: 1089-1093, 1985.

5. White PC, New MI and Dupont B: Structure of human steroid 21-hydroxylase genes. Proc Natl Acad Sci USA 83: 5111-5115, 1986.

6. Bruque CD, Delea M, Fernández CS, Orza JV, Taboas M, Buzzalino N, Espeche LD, Solari A, Luccerini V, Alba L, et al: Structure-based activity prediction of CYP21A2 stability variants: A survey of available gene variations. Sci Rep 6: 39082 , 2016.

7. Lee HH: Mutational analysis of CYP21A2 gene and CYP21A1P pseudogene: Long-range PCR on genomic DNA. Methods Mol Biol 1167: 275-287, 2014.

8. Li C, Zhou X, Han W, Jiang X, Liu J, Fang L, Wang H, Guan Q, Gao L, Zhao J, et al: Identification of two novel mutations in SLC12A3 gene in two Chinese pedigrees with Gitelman syndrome and review of literature. Clin Endocrinol (Oxf) 83: 985-993, 2015

9. Menassa R, Tardy V, Despert F, Bouvattier-Morel C, Brossier JP, Cartigny M and Morel Y: p.H62L, a rare mutation of the CYP21 gene identified in two forms of 21-hydroxylase deficiency. J Clin Endocrinol Metab 93: 1901-1908, 2008.

10. Antonarakis SE: Recommendations for a nomenclature system for human gene mutations. Nomenclature Working Group. Hum Mutat 11: 1-3, 1998.

11. den Dunnen JT and Antonarakis SE: Mutation nomenclature extensions and suggestions to describe complex mutations: A discussion. Hum Mutat 15: 7-12, 2000.

12. White PC: Congenital adrenal hyperplasias. Best Pract Res Clin Endocrinol Metab 15: 17-41, 2001.

13. Bánlaki Z, Szabó JA, Szilágyi Á, Patócs A, Prohászka Z, Füst G and Doleschall M: Intraspecific evolution of human RCCX copy number variation traced by haplotypes of the CYP21A2 gene. Genome Biol Evol 5: 98-112, 2013.

14. Barbaro M, Soardi FC, Östberg LJ, Persson B, de Mello MP, Wedell $A$ and Lajic S: In vitro functional studies of rare CYP21A2 mutations and establishment of an activity gradient for nonclassic mutations improve phenotype predictions in congenital adrenal hyperplasia. Clin Endocrinol (Oxf) 82: 37-44, 2015.

15. Yu Y, Wang J, Huang X, Wang Y, Yang P, Li J, Tsuei SH, Shen Y and Fu Q: Molecular characterization of 25 Chinese pedigrees with 21-hydroxylase deficiency. Genet Test Mol Biomarkers 15: 137-142, 2011.

16. Coeli FB, Soardi FC, Bernardi RD, de Araújo M, Paulino LC, Lau IF, Petroli RJ, de Lemos-Marini SH, Baptista MT, Guerra-Júnior G and de-Mello MP: Novel deletion alleles carrying CYP21A1P/A2 chimeric genes in Brazilian patients with 21-hydroxylase deficiency. BMC Med Genet 11: 104, 2010.

17. Concolino P, Mello E, Zuppi C and Capoluongo E: Molecular diagnosis of congenital adrenal hyperplasia due to 21-hydroxylase deficiency: An update of new CYP21A2 mutations. Clin Chem Lab Med 48: 1057-1062, 2010.

18. Lee HH, Chao HT, Ng HT and Choo KB: Direct molecular diagnosis of CYP21 mutations in congenital adrenal hyperplasia. J Med Genet 33: 371-375, 1996.

19. Concolino P, Mello E, Toscano V, Ameglio F, Zuppi C and Capulongo E: Multiplex ligation-dependent probe amplification (MLPA) assay for the detection of CYP21A2 gene deletions/duplications in congenital adrenal hyperplasia: First technical report. Clin Chim Acta 402: 164-170, 2009.

20. White PC, Vitek A, Dupont B and New MI: Characterization of frequent deletions causing steroid 21-hydroxylase deficiency. Proc Natl Acad Sci USA 85: 4436-4440, 1988.

21. Parajes S, Quinterio C, Domínguez F and Loidi L: A simple and robust quantitative PCR assay to determine CYP21A2 gene dose in the diagnosis of 21-hydroxylase deficiency. Clin Chem 53: 1577-1584, 2007.

22. Lee HH, Lee YJ and Lin CY: PCR-based detection of the CYP21 deletion and TNXA/TNXB hybrid in the RCCX module. Genomics 83: 944-950, 2004. 
23. Krone N, Reisch N, Idkowiak J, Dhir V, Ivison HE, Hughes BA, Rose IT, O'Neil DM, Vijzelaar R, Smith MJ, et al: Genotype-phenotype analysis in congenital adrenal hyperplasia due to P450 oxidoreductase deficiency. J Clin Endocrinol Metab 97: E257-E267, 2012.

24. Concolino P, Mello E, Patrosso MC, Penco S and Zuppi C: p.H282N and p.Y191H: 2 novel CYP21A2 mutations in Italian congenital adrenal hyperplasia patients. Metabolism 61: 519-524, 2012.

25. Baumgartner-Parzer SM, Fischer G and Vierhapper H: Predisposition for de novo gene aberrations in the offspring of mothers with a duplicated CYP21A2 gene. J Clin Endocrinol Metab 92: 1164-1167, 2007.

26. Leccese A, Longo V, Dimatteo C, De Girolamo G, Trunzo R, D'Andrea G, Bafunno V, Margaglione M and Santacroce R: Lack of genotype-phenotype correlation in congenital adrenal hyperplasia due to a CYP21A2-like gene. Clin Chim Acta 437: 48-51, 2014.
27. Anastasovska V and Kocova M: Genotype-phenotype correlation in CAH patients with severe CYP21A2 point mutations in the Republic of Macedonia. J Pediatr Endocrinol Metab 23. 921-926, 2010.

28. New MI, Abraham M, Gonzalez B, Dumic M, Razzaghy-Azar M, Chitayat D, Sun L, Zaidi M, Wilson RC and Yuen T: Genotype-phenotype correlation in 1,507 families with congenital adrenal hyperplasia owing to 21-hydroxylase deficiency. Proc Natl Acad Sci USA 110: 2611-2616, 2013.

29. Janjanin N, Dumic M, Skrabic V, Kusec V, Grubic Z and Spehar Uroic A: Five patients with congenital adrenal hyperplasia due to 21-hydroxylase deficiency (one with associated neuroblastoma) discovered in three generations of one family. Horm Res 67: 111-116, 2007.

This work is licensed under a Creative Commons Attribution-NonCommercial-NoDerivatives 4.0 International (CC BY-NC-ND 4.0) License. 